\title{
ABUNDÂNCIA, RIQUEZA E DIVERSIDADE DE ABELHAS EUGLOSSINAE (HYMENOPTERA, APIDAE) EM FLORESTAS CONTÍNUAS DE TERRA FIRME NA AMAZÔNIA CENTRAL, BRASIL
}

\author{
Marcio Luiz de Oliveira ${ }^{1}$ \\ Lúcio Antônio de Oliveira Campos ${ }^{2}$
}

\begin{abstract}
ABINI)ANCF. RICHNESS AND DIVERSITY OF ELGIOSSINAE BEFS

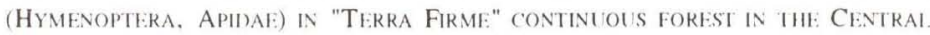
AMAZON. BRAZ1L. The fauna of Euglossinae bees were studied in two areas of "terra firme" continuous forest in the Central Amazon. Brazil. During one year (september/1989-august/1990) the collections were done fortnightly, using traps with eight kinds of scents haits. The traps were placed in the understory and in the tree crowns. The continuous forest of "terra firme" near Manaus are among those with the highest species richness of Euglossinae in the Neotropics. The species richness was higher than that found in other studies carried out in Brazil also. One study area had higher species richness and ahundance. while species diversity was higher in another one. The diversity was different significally between the two areas.

KEY WORDS. Apidae, Euglossinae. Abundance. Amazon. diversity
\end{abstract}

As abelhas da subfamília Euglossinae apresentam comumente cores metálicas, principalmente verde, e os machos são freqüentemente encontrados visitando orquídeas. O grupo está amplamente distribuído pela região neotropical, indo desde o México até a Argentina (Dressler 1982) e possui seis gêneros e aproximadamente 200 espécies (Jesus S. Moure, comunicação pessoal).

Os Euglossinae, de um modo geral, são abelhas rohustas e com grande capacidade de vôo (JANZEN 1971), por isso a maioria dos autores que as estudam têm assumido que coletas feitas num único sítio representam a fauna kocal. ARMBRUSTER (1993) argumenta que embora a floresta tropical pareça homogênea aos olhos humanos, do ponto de vista de uma abelha forrageira ela é um mosaico de diversos microhabitats determinado pela distribuição e pelo padrão de floração de diversas espécies de plantas e no caso dos Euglossinae, pelas fontes de fragrâncias. Para BECKER et al. (1991) torna-se difícil caracterizar a fauna de Euglossinae de uma floresta fazendo-se amostragem num único local.

O objetivo deste trabalho foi conhecer a abundância, riqueza e diversidade de abelhas Euglossinae em florestas contínuas de terra firme pouco alteradas, na região de Manaus, Amazônia Central, fazendo-se amostragem em dois locais.

1) Departamento de Ciêneias da Natureza. Universidade Federal de Acre. 69915-90) Rio Braneo, Acre. Brasil.

2) Departamento de Biologia Geral. Universidade Federal de Viçosa. 36570-(0)0 Viçosa. Minas Gerais. Brasil. 


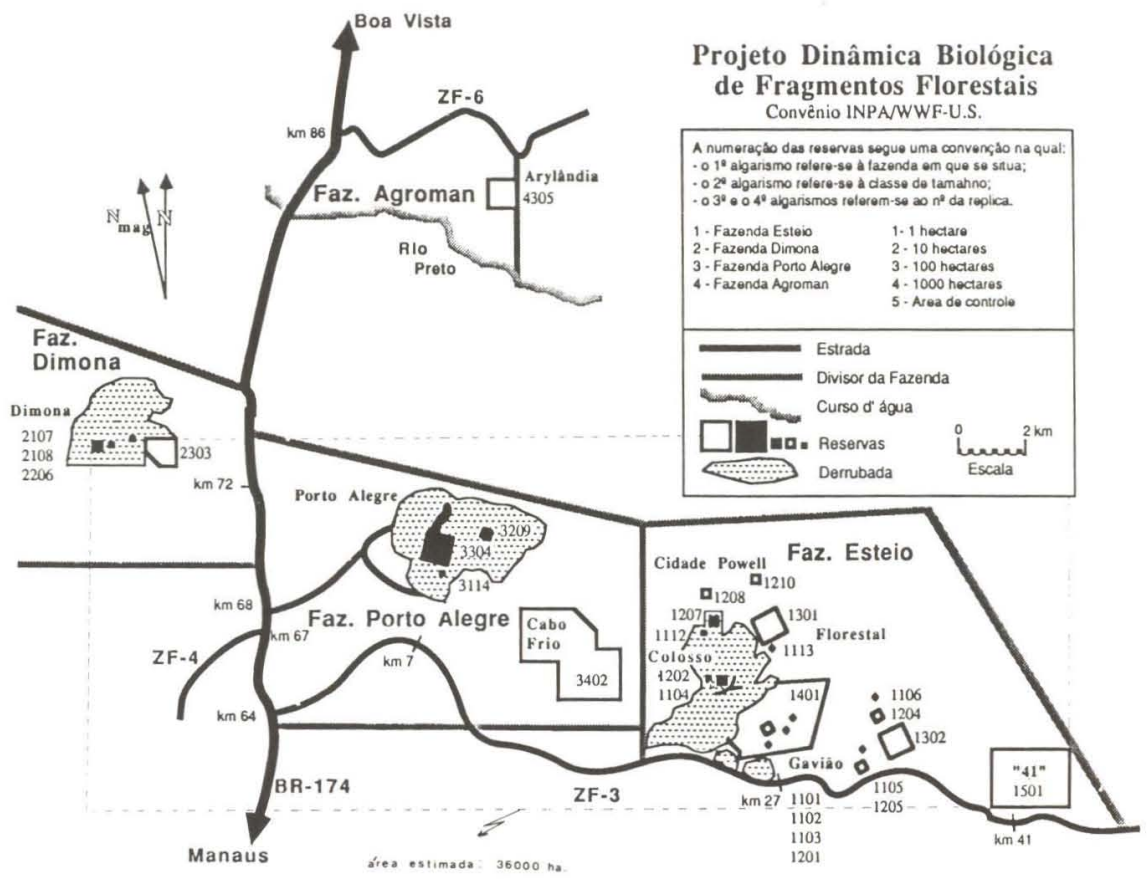

Fig. 1. Localização das duas áreas estudadas: Área $1401\left(2^{\circ} 24^{\prime} 36^{\prime \prime} \mathrm{S}, 59^{\circ} 52^{\prime} 3^{\prime \prime} \mathrm{W}\right)$ e Área $1501\left(2^{\circ} 24^{\prime} 26^{\prime \prime S}, 59^{\circ} 43^{\prime} 40^{\prime \prime} W\right)$. Setas embaixo à direita.

\section{MATERIAL E MÉTODOS}

Este estudo foi realizado em áreas sob administração do Projeto Dinâmica Biológica de Fragmentos Florestais, convênio entre o Instituto Nacional de Pesquisas da Amazônia e a Smithsonian Institution (PDBFF-INPA/SI).

As coletas foram realizadas em duas áreas de uma floresta contínua, fora dos limites das reservas de número 1401 e 1501 (numeração usada pelo PDBFF) que serão daqui em diante chamadas de Área 1401 (2²4'36"S, 59052'3"W ) e Área $1501\left(2^{\circ} 24^{\prime} 26^{\prime \prime} \mathrm{S}, 59^{\circ} 43^{\prime} 40^{\prime \prime} \mathrm{W}\right)$. Estas áreas estão situadas na estrada ZF-3, $\mathrm{Km} 27$ e 41 respectivamente, a aproximadamente $90 \mathrm{Km}$ ao norte de Manaus, ao longo da BR-174 e se encontram sob jurisdição da SUFRAMA (Superintendência da Zona Franca de Manaus), sendo que parte foi desmatada para implantação de pecuária de corte (Fig. 1). São caracterizadas como "floresta contínua tropical úmida de terra firme", com dossel atingindo em média $30-37 \mathrm{~m}$ de altura. O sub-bosque é aberto e possui um grande número de palmeiras acaules. Os solos são em geral do tipo latossolo, pobre em nutrientes e o relevo é plano-ondulado, recortado por pequenos e numerosos cursos d'água. A precipitação média anual é de $2186 \mathrm{~mm}$, com uma estação mais seca compreendida entre julho e setembro, quando a precipitação pode ser inferior a $100 \mathrm{~mm}$ em alguns meses (BIERREGAARD JR. \& LOVEIOY 1988). 
No interior de cada área foram escolhidos três locais para as coletas e em cada um deles, oito árvores, onde foram colocadas armadilhas contendo substâncias odoríferas. Em cada árvore foram instaladas duas armadilhas com uma mesma substância odorífera, uma próximo à copa (aproximadamente 10-12m) que era manejada por um sistema de carretilhas com corda e outra próximo ao solo $(0,5 \mathrm{~m})$.

Foram utilizadas armadilhas do tipo biológico da MELPAN Produtos Agrícolas, Ltda., modificadas segundo CAMPOS et cl. (1989). As substâncias odoríferas utilizadas foram acetato de benzila, benzoato de benzila, cineol, eugenol, escatol, cinamato de metila, salicilato de metila e vanilina.

As coletas foram realizadas quinzenalmente no período compreendido entre setembro de 1989 e agosto de 1990. As armadilhas eram instaladas às 7:00 horas e recolhidas 24 horas depois.

Os valores de diversidade da fauna de Euglossinae para as duas áreas, foram estimados pelo índice de Shannon (MagurRan 1988). Os valores de equitabilidade para as duas áreas foram estimados pelo índice J 'de Pielou (LUDWIG \& REYNOLDS 1988).

As abelhas foram identificadas por meio de chaves dicotômicas e por comparação com material previamente identificado e depositado na coleção entomológica da Universidade Federal de Viçosa. Posteriormente a identificação foi confirmada pelo Prof. Dr. Jesus S. Moure da Universidade Federal do Paraná.

\section{RESULTADOS}

Foram coletados nas duas áreas 2422 indivíduos pertencentes a 38 espécies. As espécies mais abundantes foram Euglossa stilbonota (785 indivíduos), $E$. chalybeata (352), E. augaspis (296), E. avicula (279) e E. crassipunctata (151), o que equivale a aproximadamente $77 \%$ da amostra.

Euglossa foi o gênero mais abundante com 28 espécies, correspondendo a cerca de $90 \%$ do total de indivíduos. Três espécies: Euglossa sp. 1, Euglossa sp. 2 e Euglossa sp. 3 ainda estão em estudo e parecem constituir espécies novas. Euglossa bidentata, E. piliventris, E. prasina e Euglossa sp. 2 além de raras, só foram encontradas na área 1501. Euglossa sp. 1, Euglossa sp. 3, E. laevecincta, e Euplusia vidua foram capturadas uma única vez (Tab. I).

A distribuição de freqüência mostra que aproximadamente $68 \%$ das espécies coletadas estão representadas por menos de 20 indivíduos (Tab. II).

A área 1501 teve o maior número de indivíduos e também o maior número de espécies. No entanto, os índices de diversidade de Shannon (H’) e de equitahilidade de Pielou (J') apresentaram maiores valores nárea 1401 (Tab. III).

\section{DISCUSSÃO}

A abundância e a riqueza faunística de abelhas Euglossinae encontradas neste trabalho são superiores às de outros levantamentos realizados no Brasil (Tab. IV) e talvez possam ser explicados, pelo menos em parte, pelo fato deste ter sido o experimento yue empregou o maior número de substâncias odoríferas, em arma- 
Tabela I. Abelhas Euglossinae coletadas em duas áreas de mata contínua de terra firme na Amazônia Central.

\begin{tabular}{|c|c|c|c|c|}
\hline Espécie & Área 1401 & Área 1501 & Porcentagem & Total \\
\hline Euglossa analis Westwood, 1840 & 3 & 2 & 0,21 & 5 \\
\hline Euglossa augaspis Dressler, 1982 & 153 & 143 & 12,22 & 296 \\
\hline Euglossa avicula Dressler, 1982 & 136 & 143 & 11,52 & 279 \\
\hline Euglossa bidentata Dressler, 1982 & - & 3 & 0,12 & 3 \\
\hline Euglossa chalybeata Friese, 1925 & 121 & 231 & 14,53 & 352 \\
\hline Euglossa cognata Moure, 1970 & 2 & 14 & 0,66 & 16 \\
\hline Euglossa crassipunctata Moure, 1968 & 64 & 87 & 6,23 & 151 \\
\hline Euglossa decorata Smith, 1854 & 14 & 4 & 0,74 & 18 \\
\hline Euglossa gaianii Dressler, 1982 & 8 & 8 & 0,66 & 16 \\
\hline Euglossa ignita Smith, 1854 & 14 & 10 & 0,99 & 24 \\
\hline Euglossa imperialis Cockerell, 1922 & 2 & 10 & 0,49 & 12 \\
\hline Euglossa intersecta Latreille, 1938 & 2 & 8 & 0,41 & 10 \\
\hline Euglossa ioprosopa Dressler, 1982 & 4 & 5 & 0.37 & 9 \\
\hline Euglossa iopyrrha Dressler, 1982 & 3 & 9 & 0,49 & 12 \\
\hline Euglossa laevicincta Dressler, 1982 & - & 1 & 0,04 & 1 \\
\hline Euglossa mixta Friese, 1899 & 27 & 40 & 2,77 & 67 \\
\hline Euglossa modestior Dressler, 1982 & 7 & 2 & 0,37 & 9 \\
\hline Euglossa mourei Dressler, 1982 & 23 & 19 & 1,73 & 42 \\
\hline Euglossa parvula Dressler, 1982 & 12 & 20 & 1,32 & 32 \\
\hline Euglossa piliventris Guérin, 1845 & - & 2 & 0,08 & 2 \\
\hline Euglossa prasina Dressler, 1982 & & 2 & 0,08 & 2 \\
\hline Euglossa retroviridis Dressler, 1982 & 9 & 10 & 0.78 & 19 \\
\hline Euglossa stilbonota Dressler, 1982 & 311 & 474 & 32,41 & 785 \\
\hline Euglossa viridifrons Dressler, 1982 & 8 & 8 & 0,66 & 16 \\
\hline Euglossa viridis (Perty, 1833) & 2 & 3 & 0,21 & 5 \\
\hline Euglossa sp. 1 & & 3 & 0,12 & 3 \\
\hline Euglossa sp. 2 & 2 & $\cdot$ & 0,08 & 2 \\
\hline Euglossa sp. 3 & & 1 & 0,04 & 1 \\
\hline Eulaema bombiformis (Packard, 1869) & 6 & 7 & 0,54 & 13 \\
\hline Eulaema meriana (Olivier, 1789) & 42 & 38 & 3,30 & 80 \\
\hline Eulaema cingulata (Fabricius, 1804) & 5 & 1 & 0,25 & 6 \\
\hline Eulaema mocsaryi (Friese, 1899) & 17 & 11 & 1,16 & 28 \\
\hline Eufriesea pulchra Smith, 1854 & 2 & 1 & 0,12 & 3 \\
\hline Euplusia ornata (Mocsáry, 1896) & 5 & 4 & 0,37 & 9 \\
\hline Euplusia vidua Moure, 1976 & 1 & - & 0,04 & 1 \\
\hline Exaerete frontalis (Guérin, 1845) & 50 & 30 & 3,30 & 80 \\
\hline Exaerete smaragdina (Guérin, 1845) & 5 & 5 & 0,41 & 10 \\
\hline Exaerete trochantherica (Friese, 1900) & 1 & 2 & 0,12 & 3 \\
\hline Total de individuos & 1061 & 1361 & & 2422 \\
\hline Total de espécies & 32 & 36 & & 38 \\
\hline
\end{tabular}


dilhas eficientes e colocadas em dois estratos da floresta.

Se somadas às 38 espécies deste trabalho, outras coletadas anteriormente na mesma região como Euglossa platymera e Eufiriesea surinamensis (ver KIMSEY \& Dressler 1986); Eufriesea lamiventris, E. xantha e E. purpurata (Powell \& POWELL 1987); Euglosia if. amazonica (BECKER et al. 1991); Euglosisa of. securigera e E. liopoda (MORATo et al. 1992); Euplusia theresiae (MORATO 1993), o número de espécies chega a 47. Isto numa área de aproximadamente 36000 ha, considerando tlorestas contínuas, fragmentos de florestas e áreas desmatadas.

\begin{tabular}{|c|c|c|}
\hline Número de indivíduos & Número de espécies & Porcentagem \\
\hline $01-20$ & 26 & 68,42 \\
\hline $21-40$ & 3 & 7,89 \\
\hline $41-60$ & 1 & 2,63 \\
\hline $61-80$ & 3 & 7,89 \\
\hline $101-200$ & 1 & 2,63 \\
\hline $201-300$ & 2 & 5,26 \\
\hline $301-400$ & 1 & 2,63 \\
\hline $700-800$ & 1 & 2,63 \\
\hline
\end{tabular}

Tabela III. Índice de diversidade de Shannon $\left(\mathrm{H}^{\prime}\right)$ e de equitabilidade de Pielou ( $\left.\mathrm{J}^{\prime}\right)$ para duas áreas de mata contínua de terra firme na Amazônia Central.

\begin{tabular}{ccc}
\hline Área & $H^{\prime}$ & $J^{\prime}$ \\
\hline 1401 & 2,389 & 0,689 \\
1501 & 2,257 & 0,630 \\
\hline
\end{tabular}

*. Todos os valores de diversidade foram significativamente diferentes $(\mathrm{t}=2,556 ; \mathrm{G}$. L. $=1918 ; \mathrm{p}=0,005)$.

Euglossa stilbonota e E. chalybeata também foram as espécies mais comuns nos levantamentos de Becker et al. (1991) e no de MORATo et al. (1992). Contudo, no de Braga (1976) foram Euglossa ignita e E. stilbonota e no de Powell \& Powell (1987) foram E. chalyheata e Eulaema meriana. É bastante provável que o baixo esforço de coleta, de apenas quatro dias, realizado por BRAGA (1976) aliado ao fato dele ter coletado em áreas de florestas de terra firme, campina e campinarana tenha provocado resultados yue não coincidem com os dos demais autores. Powell \& Powell (1987) por sua vez, apesar de terem realizado seu trabalho no mesmo local que este, só o fizeram durante a estação seca.

Por outro lado, Eufivesea laniventrix, E. xamtha e E. purpurata coletadas por Powell \& Powell (1987) com puçà, em salicilato de metila, não foram 
coletadas no presente trabalho. É possível que o uso de armadilhas, nesse caso, tenha impedido a captura destas espécies. Além disso, Eufiriesea purpurata, coletada por Powell \& Powell (1987), Euglossa of securigera e E. liopoda, coletadas por MORATO et al. (1992), ocorreram principalmente em áreas de derrubada e borda de mata, ambientes em que não se fez coletas. Euglossa ignita foi a espécie mais comum nos três ambientes estudados por BRAGA (1976). Os resultados aqui obtidos mostram que as florestas de terra firme próximas a Manaus estão entre as mais ricas em Euglossinae (Tab. V).

Tabela IV. Levantamentos de abelhas Euglossinae realizados no Brasil utilizando-se substâncias odoríferas (S.O).

\begin{tabular}{lcccr}
\hline \multicolumn{1}{c}{ Autores } & Estado & Número de S.O & Individuos & Espécies \\
\hline Wittmann et al. 1988 & RS & 3 & 639 & 5 \\
Rebelo \& Garófalo 1991 & SP & 3 & 892 & 8 \\
Abrantes 1990 & MG & 4 & 896 & 11 \\
Raw 1989 & $\mathrm{BA}$ & 5 & 1285 & 5 \\
Aguilar 1990 & $\mathrm{BA}$ & 5 & 280 & 9 \\
Gomes 1991 & $\mathrm{MA}$ & 4 & 1728 & 13 \\
Braga 1976 & $\mathrm{AM}$ & 4 & 76 & 10 \\
Powell \& Powell 1987 & $\mathrm{AM}^{*}$ & 3 & 992 & (2) 15 \\
Becker et al. 1991 & $\mathrm{AM}^{*}$ & 3 & 290 & (1) 16 \\
Morato et al. 1992 & $\mathrm{AM}^{* *}$ & 4 & 1242 & (2) 27 \\
Oliveira \& Campos & $\mathrm{AM}^{* *}$ & 8 & 2422 & 38 \\
\hline
\end{tabular}

${ }^{*}$. Áreas do PDBFF; ${ }^{*}$. áreas adjacentes ao PDBFF. Entre parêntesis o número de espécies não compartilhadas com este trabalho.

PEARSON \& DRessler (1985) encontraram no Peru, riqueza de espécies de Euglossinae semelhante a de Manaus e Belém no Brasil, Panamá e Costa Rica e formularam as seguintes hipóteses: 1) muitas espécies de Euglossinae ainda permanecem desconhecidas na Amazônia e esta aparente semelhança poderia ser explicada pelo fato de terem sido utilizadas as mesmas iscas nesses locais; 2) esta semelhança no seria por causa das iscas mas pelo fato que, Euglossinae e provavelmente outros grupos foram relativamente não afetados pelos eventos de isolamento e especiação ocorridos no refúgios florestais durante o Pleistoceno. Esses mesmos autores, baseados em outros trabalhos que empregaram um grande número de iscas e levando em conta ainda a resistência fisiológica e grande mobilidade dos Euglossinae, sugerem como mais provável a segunda hipótese. De fato, apesar de muitas iscas terem sido utilizadas nos diversos trabalhos realizados na Amazônia, as abelhas parecem preferir um número reduzido delas, visitando pouco ou nada as demais. Apesar de PEARSON \& DRESSLER (1985) citarem 38 espécies para aquela região situada no leste do Peru, apresentam uma lista contendo apenas 28. Destas, apenas Euglossa perviridis, exclusiva do Peru e Eg. despecta do Peru e Panamá, não ocorrem na Amazônia brasileira. Uma outra espécie citada, Exaerete dentata, também não tem sido encontrada na Amazônia brasileira, mas fora de seus limites, em pleno domínio das Caatingas, no Piauí (ver KIMSEY \& 
Tabela V. Levantamentos de abelhas Euglossinae na Região Neotropical utilizando-se substâncias odoriferas (S.O).

\begin{tabular}{|c|c|c|c|c|}
\hline Autores & País & Número de S.O & Individuos & Espécies \\
\hline \multirow[t]{18}{*}{ Dodson et al. 1969} & México (L) & 5 & 203 & 9 \\
\hline & México (0) & & 119 & 5 \\
\hline & México (S) & & 605 & 9 \\
\hline & Guatemala & & 68 & 6 \\
\hline & El Salvador & & 31 & 6 \\
\hline & Honduras & & 28 & 8 \\
\hline & Nicarágua & & 73 & 4 \\
\hline & Costa Rica (N) & & 88 & 17 \\
\hline & Costa Rica (S) & & 93 & 20 \\
\hline & Panamá & & 927 & 48 \\
\hline & Colômbia (L) & & 160 & 42 \\
\hline & Colômbia (O) & & 29 & 6 \\
\hline & Equador (L) & & 427 & 18 \\
\hline & Equador $(0)$ & & 121 & 13 \\
\hline & Venezuela (L) & & 89 & 18 \\
\hline & Venezuela (0) & & 173 & 15 \\
\hline & Guianas & & 107 & 4 \\
\hline & Trinidad & & 244 & 13 \\
\hline \multirow[t]{4}{*}{ Ricklefs et al. 1969} & Panamá & 1 & 117 & 15 \\
\hline & Panamá & & 189 & 22 \\
\hline & Panamá & & 173 & 11 \\
\hline & Panamá & & 144 & 15 \\
\hline \multirow[t]{2}{*}{ Bennett 1972} & Honduras & 6 & 142 & 10 \\
\hline & Honduras & 14 & 147 & 13 \\
\hline Williams \& Dodson 1972 & Guiana Inglesa & 23 & 713 & 71 \\
\hline Janzen 1981 & Costa Rica & 5 & 1282 & 4 \\
\hline \multirow{3}{*}{ Janzen et al. 1982} & Costa Rica & 5 & 961 & 27 \\
\hline & Costa Rica & 5 & 720 & 18 \\
\hline & Costa Rica & 5 & 480 & 13 \\
\hline \multirow[t]{4}{*}{ Ackerman 1983a } & Panamá & 16 & 21842 & 44 \\
\hline & Panamá & 16 & 4787 & 41 \\
\hline & Panamá & 8 & 4902 & 35 \\
\hline & Panamá & 16 & 5552 & 44 \\
\hline Pearson \& Dressler 1985 & Peru & 20 & 2917 & 38 \\
\hline Folsom 1985 & Colômbia & 2 & 2339 & 17 \\
\hline Roubik \& Ackerman 1987 & Panamá & 3 & 20000 & 51 \\
\hline Whitten et al. 1988 & $\begin{array}{l}\text { México, } \\
\text { Panamá e Peru }\end{array}$ & 1 & 123 & 9 \\
\hline Ackerman 1989 & Panamá & 16 & 27874 & 53 \\
\hline Gonzalez \& Gaiani 1989 & Venezuela & 1 & 69 & 16 \\
\hline Gonzalez \& Gaiani 1991 & Venezuela & 1 & 26 & 6 \\
\hline
\end{tabular}

*. (L) Leste, (O) Oeste, (S) Sul, (N) Norte. 
DRESSler 1986). De acordo com os nossos resultados, a região leste do Peru e as florestas de terra firme no norte de Manaus, apesar de serem tidas como refúgios florestais pleistocênicos (PRANCE 1973, 1982), possue m uma fauna de Euglossinae muito semelhante, ao contário do que se esperaria, o que parece confirmar a segunda hipótese levantada por PEARSON \& DRESSLER (1985).

É possível que as diferenças observadas nas populações de Euglossinae reflitam diferenças não detectadas nas composições florísticas das duas áreas estudadas em Manaus, especialmente no que se refere às plantas que produzem substâncias atrativas, das quais as mais comuns pertencem à família Orchidaceae, que por serem epífitas, são difíceis de serem observadas na floresta. Outros fatores, como presença de parasitas de ninho, diferenças no microclima ou fatores que afetem a volatilização das substâncias odoríferas (FOLSOM 1985), também podem responder por parte das diferenças observadas.

É preciso considerar ainda que as abundâncias de espécies aqui encontradas dizem respeito somente à abelhas Euglossinae que são atraídas pelas substâncias odoríferas utilizadas. Sabe-se, por exemplo, que Eufriesea purpurata é hastante comum na região, mas não tem sido capturada com as iscas utilizadas até então, mas já foi atraída pelo inseticida D.D.T. (RoberTs et al. 1982). Por outro lado, a descoberta e a utilização de novas iscas pode facilitar a coleta de espécies desconhecidas. Outro fator que deve ser considerado, é que as próprias armadilhas, apesar de eficientes, podem não capturar algumas espécies existentes na região.

Apesar das duas áreas estarem situadas numa floresta contínua, afastadas entre si por aproximadamente $9 \mathrm{~km}$ e, à primeira vista, serem hastante semelhantes na sua composição florística, o índice de diversidade de Shannon ( $\left.H^{\prime}\right)$ e também o de equitabilidade de Pielou (J') foram maiores para a Área 1401. É possível que esta distância seja uma barreira pelo menos para algumas espécies, ocasionando tal resultado. Embora se divulgue que os Euglossinae possuam grande capacidade de vôo e possam cobrir ğrandes distâncias (JANZEN 1971), trabalhos realizados na região de Manaus mostraram que quatro espécies de Euglossinae não cruzaram os 100 metros de pastagem que separava as matas contínuas dos fragmentos de mata (Powell \& Powell 1987). BeCKer et al. (1991), por sua vez, acham que a abundância de abelhas pode variar completamente em uma pequena escala espacial em resposta a fatores desconhecidos, já que em seu trabalho houve diferenças substanciais entre sítios separados por apenas 300 a 700 metros e amostrados no mesmo dia.

Ao que tudo indica, a fauna de Euglossinae é bastante diferente de um lugar para outro numa floresta tropical de terra firme, por mais semelhantes que eles se pareçam. Assim, é hem provável que muitas espécies de Euglossinae desapareçam, caso não se leve em consideração estas diferenças, na hora de se planejar futuras áreas de conservação.

AGRADECIMENTOS. Ao CNPy pela bolsa de mestrado concedida. Ao PDBFF (INPA/ Smithsonian Institution) pelo financiamento do trabalho, esta é a publiçação no. 147 do PDBFF. Ao Prof. Dr. Padre Jesus Santiago Moure pela identificação das espécies. 


\section{REFERÊNCIAS BIBLIOGRÁFICAS}

ARMBRUSTER, W.S. 1993. Within-habitat heterogeneity in baiting samples of male Euglossine bees: Possibles causes and implications. Biotropica 25 (1): 122-128.

Becker, P.; J.S. Moure \& F.J.A. Peralta. 1991. More about euglossine bees in amazonian forest fragments. Biotropica 23 (4b): 586-591.

Bierregaard JR., R.O. \& T.E. Lovejoy. 1988. Birds in Amazonian forest fragments: effects of insularization, vol. 2, p. 1564-1579. In: H. QUELLET (ed.). Acta XIX Cong. Int. Ornith. Otawa, Univ. of Otawa Press, 1409p.

BRaga, P.I.S. 1976. Atração de abelhas polinizadoras de Orchidacede com auxílio de iscas-odores na campina, campinarana e floresta tropical úmida da região de Manaus. Ciência e Cultura 28 (7): 767-773.

Campos, L.A.O.; F.A. Silveira; M.L. Oliveira; C.V.M. Abrantes; E.F. MORATO \& G.A.R. MElo. 1989. Utilização de armadilhas para a captura de machos de Euglossini (Hym., Apoidea). Revta bras. Zool. 6 (4): 621-626.

Dressler, R.L. 1982. Biology of the orchid bees (Euglossini). Ann. Rev. Ecol. Syst. 13: 373-394.

Folsom, J.P. 1985. Dos nuevas tecnicas para capturar y marcar abejas machos de la tribu Euglossini (Hymenoptera, Apidae). Actualidades Biológicas 14 (51): 20-25.

JanZEN, D.H. 1971. Euglossine bees as long-distance pollinators of tropical plants. Science 171: 203-205.

Kimsey, L.S. \& R.L. Dressler. 1986. Synonymic species list of Euglossini. Pan-Pac. Ent. 62 (3): 229-236.

LudwiG, J.A. \& J.F. REYNOLDS. 1988. Statistical ecology: A primer on methods and computing. New York, John Wiley \& Sons, 337p.

Magurran, A.E. 1988. Ecological diversity and its measurement. New Jersey, Princeton University Press, Princeton, 179p.

Morato, E.F. 1993. Efeitos da fragmentação florestal sobre vespas e abelhas solitárias em uma área da Amazônia Central. Dissertação de mestrado, não publicada, Universidade Federal de Viçosa, Departamento de Biologia Animal. Viçosa, 105p.

Morato, E.F.; L.A.O. Campos \& J.S. Moure. 1992. Abelhas Euglosisini (Hymenoptera, Apidae) coletadas na Amazônia Central. Revta bras. Ent. 36 (4): 767-771.

Pearson, D.L. \& R.L. Dressler. 1985. Two-year study of male orchid bee (Hymenoptera, Apidae, Euglossini) attraction to chemical baits in lowland south-eastern Peru. Jour. Trop. Ecol. 1: 37-54.

Powell, A.H. \& G.N.V. Powell. 1987. Population dynamics of male euglossine bees in amazonian forest fragments. Biotropica 19 (2): 176-179.

Prance, G.T. 1973. Phytogeographic support for the theory of Pleistocen forest refuges in the Amazon Basin, based on evidence from distribution patterns in Caryocaraceae, Dichapetalaceae and Lecythidaceae. Acta Amazonica 3: 5-28. 
Prance, G.T. 1982. A review of the phytogeographic evidences for pleistocene climate changes. Ann. Missouri Bot. Gard. 69: 594-624.

Roberts, D.R.; W.D. Alecrim; J.M. Heller; S.R. EhrhardT \& J.B. Lima. 1982. Male Eufriesia purpurata, a DDT-collecting euglossine bee in Brazil. Nature 297: 62-63. 